\title{
Genetic Variants Contributing to Early Recurrent Pregnancy Loss Etiology Identified by Sequencing Approaches
}

\author{
Paula Quintero-Ronderos, MD' and Paul Laissue, MD, PhD'
}

\begin{abstract}
Recurrent pregnancy loss (RPL) affects up to $5 \%$ of couples. It is believed that genetic factors contribute to the disease's etiology and pathophysiology. Hundreds of genes represent coherent RPL candidates due to mammalian implantation's inherent complexity. Sanger sequencing (direct sequencing) of candidate genes has identified potential RPL causative genes (and variants), including those regulating embryo implantation and pregnancy maintenance. Although this approach is a reliable technique, the simultaneous analysis of large genomic regions is challenging. Next-generation sequencing (NGS) technology has thus emerged as a useful alternative for determining genetic variants and transcriptomic disturbances contributing to monogenic and polygenic diseases pathogenesis. However, interpreting results remains challenging as NGS experiments provide an enormous amount of complex data. The molecular aspects of specific diseases must be fully understood for accurate interpretation of NGS data. This review was thus aimed at describing (for the first time) the most relevant studies involving Sanger and NGS sequencing, leading to the description of variants related to RPL pathogenesis. Successful RPL-related NGS initiatives (including RNAseq-based studies) and future challenges are discussed. We consider that the information given here should be useful for clinicians, scientists, and students to enable a better understanding of RPL etiology. It may also provide a basis for the development of diagnostic/prognostic approaches contributing toward translational medicine.
\end{abstract}

\section{Keywords}

recurrent pregnancy loss, next generation sequencing, molecular implantation, miscarriage

\section{Introduction}

Human infertility is a frequently occurring disease, affecting more than 50 to 80 million couples worldwide. ${ }^{1,2}$ Despite advances in diagnosis and treatment, the disease etiology remains unexplained in more than $30 \%$ of cases, strongly suggesting the involvement of genetic, epigenetic, and environmental factors. Female infertility-contributing dysfunctions are related to tubal disease, ovulation disturbances, and endometrial pathologies, including miscarriage. ${ }^{3,4}$ The latter (ie, pregnancy loss up to 24 weeks' gestation) has been associated with various etiologies, such as thrombophilic disorders, anatomic malformations, infectious agents, systemic pathologies, and endocrine, immunological, and genetic anomalies. Fetal chromosomal abnormalities (such as sporadic aneuploidies) are the commonest cause of miscarriage, accounting for up to 50\% of cases. However, fetal aneuploidy incidence in recurrent pregnancy loss (RPL) decreases with an increased amount of miscarriages. Other rare fetal chromosomal abnormalities, such as translocations and copy number variations (CNVs), are also associated with the possible recurrent risk of miscarriage. ${ }^{5-7}$ RPL (defined as $2 / 3$ consecutive miscarriages) affects up to $5 \%$ of couples. ${ }^{8,9}$ It has been proposed that genetic factors contribute to the disease etiology and physiopathology, mainly because RPL patients' siblings have higher miscarriage frequency than women from control populations. ${ }^{10-13}$

Different approaches have been adopted for exploring embryo implantation disturbances and RPL's genetic origin, such as genetic linkage analysis, DNA methylation status determination, and candidate gene genotyping. Classical linkage analysis used with affected families has been particularly challenging due to the rarity of large pedigrees being affected by reproductive disorders. Indeed, mutations leading to fertility disturbances are usually under strong negative selection. Genome-wide association study approaches (based on genome-scale polymorphic marker genotyping) have enabled

\footnotetext{
' Center For Research in Genetics and Genomics (CIGGUR), GENIUROS Research Group, School of Medicine and Health Sciences, Universidad del Rosario, Bogotá, Colombia
}

\section{Corresponding Author:}

Paul Laissue, Centro de Investigación en Genética y Genómica (CIGGUR), GENIUROS Research Group, School of Medicine and Health Sciences, Universidad del Rosario, Carrera 24 N ${ }^{\circ}$ 63C-69, Bogotá I 100100 , Colombia. Email: paul.laissue@urosario.edu.co 
several loci to be mapped (3p14.2, 9p22.1, 1q13.4, 6q16.3, 6q27, 9q33.1, and Xp22.1) and potentially RPL phenotyperelated genes to be proposed (eg, HLAs, FHIT, FAM154A, $P D E 2 A$, and GRIK2) ${ }^{12,14,15}$ It is worth noting that quantitative trait loci (QTL) mapping, using the interspecific recombinant congenic strain mouse model, has led to identifying embryonic resorption-related regions which are analogous to the human RPL phenotype. ${ }^{16-19}$ Genotyping human candidate genes located in these chromosomal regions has enabled identifying mutations in alkaline phosphatase placental (ALPP) and forkhead box D1 (FOXD1) which lead to major functional disturbances and contribute to the RPL phenotype. ${ }^{19,20}$

Sanger sequencing (direct sequencing) of candidate genes has also been used for identifying potential RPL causative genes (and variants), including those regulating embryo implantation and pregnancy maintenance. Although direct sequencing is a reliable technique, the simultaneous analysis of large genomic regions is challenging. Next-generation sequencing (NGS) has thus emerged as a useful alternative for determining genetic variants and transcriptomic disturbances contributing to monogenic and polygenic disease pathogenesis. ${ }^{21-23}$ Next-generation sequencing has been used in reproductive medicine for studying several female pathological conditions, such as primary ovarian insufficiency, preeclampsia, and RPL. ${ }^{24-34}$ However, interpreting results is challenging as NGS experiments provide an enormous amount of complex data, especially regarding polygenic diseases. Furthermore, specific diseases' molecular aspects must be fully understood for accurately designing and interpreting NGS experiments. Next-generation sequencing has recently been described as a useful tool for discovering new variants which are potentially related to RPL pathogenesis. After their in vitro validation, RPL candidate variants found by this approach can be considered molecular biomarkers for diagnostic/prognostic purposes. This review has thus been aimed at describing the most relevant studies involving Sanger sequencing leading to the description of variants potentially related to RPL pathogenesis.

We have included information on studies specifically using this sequencing methodology as their main technique for identifying variants potentially related to the phenotype. It should be noted that such reports have frequently been based on the candidate gene approach. Association and case-control studies have also been undertaken, most having emphasized statistical linking between variants and the phenotype. Such variants have been reported in some cases as determining a higher risk of suffering RPL and, in rare cases, have been validated by functional tests. Nevertheless, these variants constitute robust candidates for future studies and are potentially useful clinical biomarkers. Successful RPL-related NGS initiatives have also been discussed.

We consider that the information given here should be useful for clinicians, scientists, and students to enable a better understanding of RPL etiology. It may also provide a basis for diagnostic approaches contributing toward translational medicine.

\section{Candidate Gene Approach Using Sanger Sequencing in RPL}

Classical Sanger sequencing has been used for accurately determining a DNA sequence from amplicons obtained by polymerase chain reaction (PCR) amplification. Although direct sequencing is a reliable technique only $\sim 600$ base pairs (bp) per reaction can be read, thereby hampering simultaneous analysis of large genomic regions. This approach has led to identify variants (eg, polymorphisms and rare variants) being found in female RPL patients in genes related to different molecular cascades and biological processes, such as the estrogen pathway, embryo-maternal immune response, apoptosis, decidualization, angiogenesis, coagulation, metabolism, and enzymatic activity. Table 1 shows the variants in RPL patients which have been screened by Sanger sequencing.

Studies using RPL patients' DNA from peripheral blood samples have found genetic variants in genes associated with embryo-maternal immune response modulation, an important step for successful implantation during the attachment phase. It should be noted that syncytiotrophoblast (ST) secretes immunosuppressive proteins such as PAI-1 and HLA-G during invasion to avoid rejection by the maternal immune system. ${ }^{35,36}$ Several studies have found associations between immunosuppressive gene variants and RPL development.

A PAI-1 screening study of the $5^{\prime}$ untranslated region (UTR) region involved direct sequencing of DNA from blood samples taken from idiopathic Indian RPL patients affected by at least 2 consecutive pregnancy losses before the 20th week of gestation. The authors found a statistical association between the c.-816 $\mathrm{A}>\mathrm{G}$ (rs1799889 4G/5G polymorphism) variant (located in the promoter region) and RPL development and implantation failure $(P=.016) .{ }^{37}$ Patients having a history of smoking or alcohol use, as well as anatomic, hormonal, chromosomal, infectious, autoimmune, and thrombotic causes were excluded from the study, as were those having had a live birth.

Evidence has also been provided of $H L A-G$ variants' genetic associations with idiopathic RPL in populations having different ethnic origins, that is, Caucasian, Indian, and EuroBrazilian. HLA-G's complete open reading frame (ORF), $5^{\prime}$ UTR, and $3^{\prime}$ UTR have been sequenced in different studies. ${ }^{38-40}$ These revealed that patients having c. $-1179 \mathrm{G}>\mathrm{A}$ (rs1233335, odds ratio [OR]: $3.8, P<.001$ ), c. ${ }^{*} 29-126 \mathrm{G}>\mathrm{A}$ (rs915670, OR: 2.45, $P=.008$ ), and c. $788 \mathrm{C}>\mathrm{A}$ (rs114252012, OR: $2.35, P=.019$ ) variants and $H L A-G$ alleles $(* 0105 \mathrm{~N}$ and $* 01013, P=.007)$ had a higher risk of developing RPL or suffering implantation failure compared to controls. The patients in those studies had 2 or more unexplained spontaneous miscarriages and no abnormalities found during gestational screening. Abnormal couple karyotype, antiphospholipid and lupus antibodies, prothrombotic and endocrine factors, hormonal levels, and uterine anatomy alterations were the exclusion criteria. ${ }^{38-41}$

Functional tests regarding $H L A-G$ variants found in Indian and Euro-Brazilian populations have elucidated HLA-G expression levels in such patients. Agrawal et al measured 
Table I. Variants Associated With RPL Using Direct Sequencing Approach.

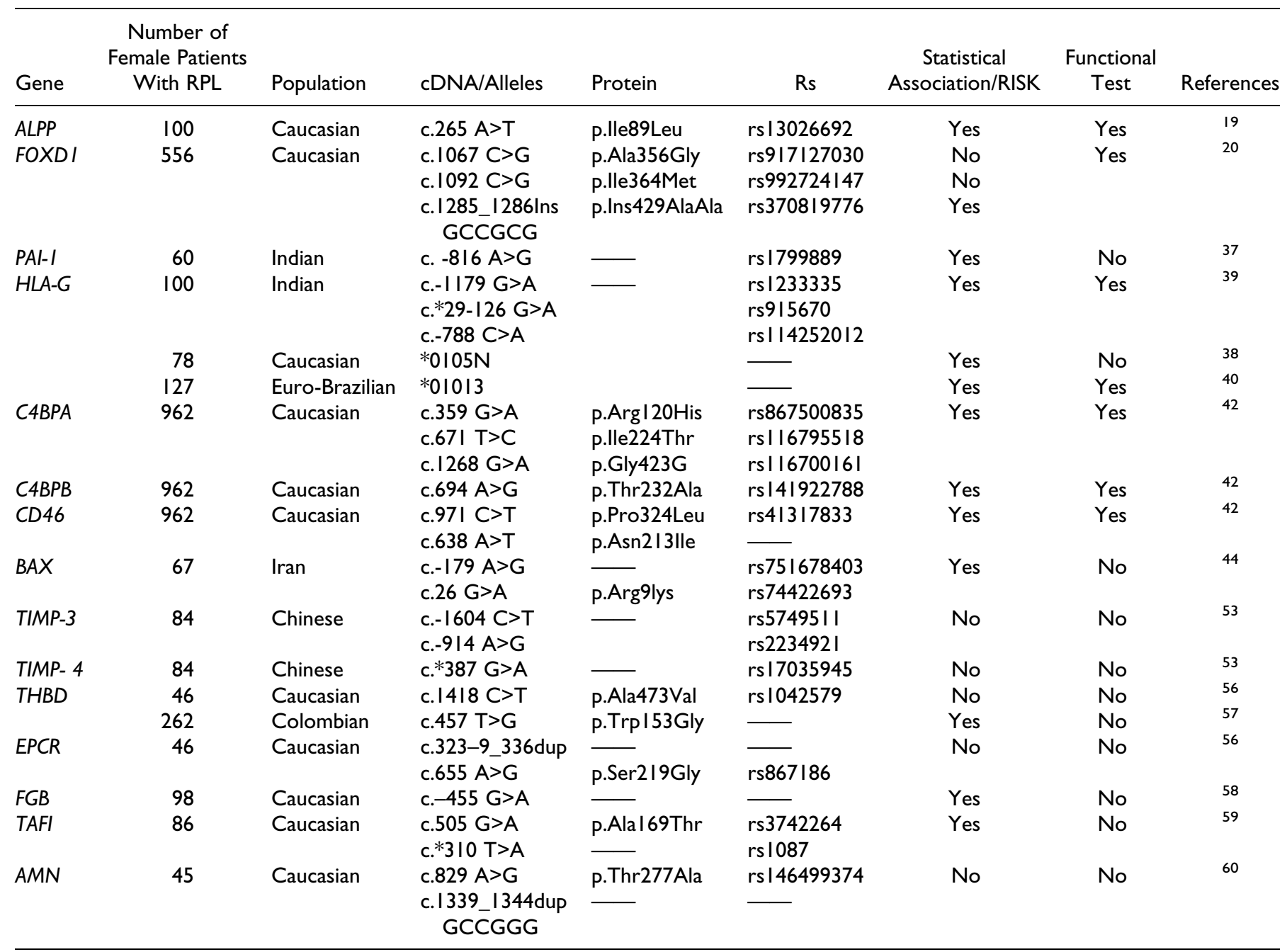

Abbreviations: ALPP, alkaline phosphatase placental; cDNA, complementary DNA; MMP, metalloproteinase; RLP, recurrent pregnancy loss.

HLA-G messenger RNA levels by quantitative PCR of blood samples from Indian RPL patients. They found a significant decrease in $H L A-G$ expression in those patients carrying the HLA-G c. $1179 \mathrm{G}>\mathrm{A}$ (rs1233335) and c.*29-126G $>\mathrm{A}$ (rs915670) variants compared to control group. ${ }^{39}$ Nardi et al used an enzyme-linked immunosorbent assay (ELISA) for measuring soluble HLA-G (sHLA-G) concentration in EuroBrazilian patients' peripheral blood; however, no significant differences were found when the patients' serum levels were compared to control group. ${ }^{40}$

Another study has reported that variants in complement and lymphocyte receptors may contribute toward RPL etiology, that is, C4BPA (c.359 G>A, p.Arg120His - rs867500835; c.671 T $>\mathrm{C}$, p.Ile224Thr - rs116795518 and c.1268 G>A, p.Gly423Glu rs116700161), $C 4 B P B$ (c.694A $>$ G, p.Thr232Ala - rs141922788), and $C D 46$ (c.971 C>T, p.Pro324Leu - rs41317833 and c.638 A > T, p.Asn213Ile). ${ }^{42}$ This study involved Caucasian women having suffered at least 3 consecutive embryonic losses. Patients having risk factors such as abnormal karyotype, infectious disease during pregnancy, metabolic, autoimmune, and endocrine diseases, and uterine anomalies were excluded from the study. Mohlin et al sequenced the complete $C 4 B P A, C 4 B P B$, and $C D 46$ ORFs and used ELISA for analyzing expression levels and C4BPA (p.Arg120His and p.Gly423Glu) and CD46 (p.Pro324Leu and p.Asn213Ile) variants' degradation activity. The authors found that the p.Gly423Glu variant mutation affected C4BPA expression level, while the C4BPA p.Arg120His variant increased C4BP ability to act as a $\mathrm{C} 4 \mathrm{~b}$ degradation cofactor $(P<.001)$. Furthermore, the C4BPA p.Arg120His variant also decreased C3b degradation levels $(P<.01)$. CD46 p.Pro324Leu and p.Asn213Ile variants' functional tests revealed a significant decrease in CD46 expression level and decreased cofactor activity regarding $\mathrm{C} 4 \mathrm{~b}(P<.001)$ and C3b degradation $(P<.01) .^{42}$

Regarding the clinical setting, a study has shown that RPL patients' apoptotic gene expression levels were higher than control group levels, thereby increasing apoptosis rate and altering blastocyst invasion. ${ }^{43}$ The $B A X$ promoter region and complete ORF were sequenced to identify variants in RPL Iranian patients. The inclusion criteria were 3 or more miscarriages before the 20th week of gestation. Patients having 
positive findings during clinical and laboratory evaluation were excluded from this study, that is, hysteroscopy, karyotyping, cervical culture, hormonal, and autoantibody levels. $B A X$ c.-179 A $>\mathrm{G}-\operatorname{rs} 751678403(P=.0128)$ and c. $26 \mathrm{G}>\mathrm{A}$, p.Arg9lys - rs74422693 $(P<.0001)$ variants had a statistical association with RPL. ${ }^{44}$

Epithelial cell apoptosis promotes trophoblast cell invasion and subsequent penetration of endometrial decidual cells by adhesion protein and extracellular matrix (ECM) degradation. Progesterone increases TIMP expression via upregulation of transforming growth factor beta 1 (TGF- $\beta$ ) and interleukin-1, thus avoiding excess extravillous ST invasion. ${ }^{45-47}$ Metalloproteinases (MMPs) have been shown to have a critical function regarding ECM degradation during ST invasion. $M M P-2$ and $M M P-9$ expression is controlled by progesterone by inhibiting SP4 and NF- $\kappa \beta$ transcription factors. ${ }^{48-52}$

Studies using Sanger sequencing have revealed genetic polymorphisms in TIMP-3 (c.-1604C $>\mathrm{T}$, rs5749511 $-P=.871$, OR $=1.074$ and c. $-914 \mathrm{~A}>\mathrm{G}-\mathrm{rs} 2234921-P=.559, \mathrm{OR}=1.659)$ and TIMP-4 (c. $* 387 \mathrm{G}>\mathrm{A}-\mathrm{rs} 17035945-P=.229, \mathrm{OR}=0.679)$. However, a statistical association between these variants and the phenotype was not clearly established in the target Chinese population, nor was the risk of developing RPL. ${ }^{53}$ These studies involved patients having suffered 3 or more unexplained RPL; patients having an abnormal karyotype, antiphospholipid syndrome (APS), uterine anomalies, endocrine dysfunction, thrombophilia, and infections were excluded.

The endometrium's stromal cells change their morphology and accumulate glycogen and lipids in their cytoplasm during the decidualization stage; these will become sources of nutrition for an implanted embryo. Decidualization also controls ST invasion and promotes placenta development. Angiogenesis and endometrial spiral artery remodeling during decidualization is a crucial step for correct embryo implantation. Such morphological changes in blood vessels include their dilatation and the apoptosis of endothelial and smooth muscle cells in the tunica media. These modifications enable the flow of oxygen and nutrients to the embryo. ${ }^{54,55}$ Coagulation proteins such as thrombomodulin (THBD) and EPCR are essential for blood flow hemostasis, thus preventing hypercoagulation states.

Various genetic association studies have found $T H B D$ and $E P C R$ variants $(T H B D$ c. $1418 \mathrm{C}>\mathrm{T}$, p.Ala473Val - rs1042579, $T H B D$ c.457T $>$ G, p.Trp153Gly, EPCR c.323-9_336dup, and EPCR c.655A $>$ G, p.Ser219Gly - rs867186) in Caucasian and Colombian RPL patients affected by 2 or more consecutive unexplained miscarriages. However, only THBD-p.Trp153Gly had a statistical association with the disease's origin $(P=$ .000009) ${ }^{56,57}$ These patients' miscarriages occurred during the first trimester of pregnancy and were negative for any of the classical risk factors (chromosome and uterine anomalies, autoimmunity, thrombophilia, infections, and endocrine diseases). The regions of interest in other coagulation genes (such as $F G B$ and $T A F I$ ) have been sequenced in a Caucasian population. Such studies have included patients having suffered 2 or more idiopathic RPL. Women having known risk factors were excluded from the studies. The $F G B$ c. $-455 \mathrm{G}>\mathrm{A}(P<$ $.05)$, TAFI c.505G $>$ A, p.Ala169Thr - rs3742264 $(P<.01$, OR: $1)$, and c. $* 310 \mathrm{~T}>\mathrm{A}-\operatorname{rs} 1087(P<.01$, OR: 1$)$ variants were found to be statistically associated with RPL women's phenotype. $^{58,59}$

Molecules (and genes) related to enzymatic activity and metabolism have been shown to play a role during implantation by regulating vitamin $\mathrm{B} 12(A M N)$ and phosphate catabolism. ALPP catalyzes phosphomonoester hydrolysis and may be involved in cell proliferation during early gestation. The $A L P P$ gene has been sequenced (11 introns and 12 exons) to identify variants associated with RPL. The c.265A $>$ T, p.Ile89Leu (rs13026692) variant was associated with both a decreased risk of miscarriage and fertilization failure $(P=.0162, \mathrm{OR}: 0.474)$ by enhancing alkaline phosphatase activity $(P=.01)$ in a target (French) Caucasian population of women having suffered unexplained primary recurrent miscarriages before the 20th week of gestation and in vitro fertilization failure. Patients having an abnormal karyotype/hormonal levels, prothrombotic factors, autoimmunity, infections, or uterine anomalies were excluded. This variant could be a predictor of implantation success due to functional test results demonstrating that phosphatase activity increased by around $30 \% .{ }^{19}$

$A M N$ variants (c.829A $>\mathrm{G}$, p.Thr277Ala - rs146499374 and c.1339_1344dup GCCGGG) have been reported in Caucasian RPL patients having suffered at least 3 consecutive miscarriages and having no history of an abnormal karyotype, uterine anomalies, infections during pregnancy, endocrine alterations, metabolic disorders (diabetes mellitus and hypertension), or autoimmunity. Patients having a history of any of these factors were excluded from the studies. No association with RPL etiology was demonstrated for $A M N$ gene sequence variants. ${ }^{60}$

A study using Sanger sequencing has found variants related to RPL and the FOXD1 transcription factor. The authors sequenced the complete FOXD1 encoding region using the DNA from peripheral blood samples taken from 556 idiopathic RPL Caucasian patients and 271 control women. ${ }^{20}$ Patients affected by unexplained primary recurrent miscarriages before the 20th week of gestation fulfilled the inclusion criteria. Women were excluded if they had an abnormal karyotype and/or hormonal levels, prothrombotic factors, autoimmunity disorders, infections, or uterine anomalies. The control group consisted of women who had had at least one live birth and no antecedents of pregnancy loss. The results revealed 10 nonsynonymous variants related to a 10.3 relative risk (confidence interval-CI: 1.4-77.2).

Gene reporter assays using the FOXD1-p.Ala356Gly and FOXD1-p.Ins429AlaAla variants revealed this transcription factor's inability to transactivate the $P G F$ promoter $(P<.01)$. $C 3$ promoter transactivation was enhanced by the FOXD1p.Ile364Met and FOXD1-p.Ins429AlaAla mutations, while C3 promoter transcription activity became decreased by the FOXD1-p.Ala356Gly mutations. These results suggested that FOXD1 mutations are a major cause of idiopathic RPL.

It should be noted that only a few of these variants have been studied by in vitro/in vivo functional tests, thereby limiting their 


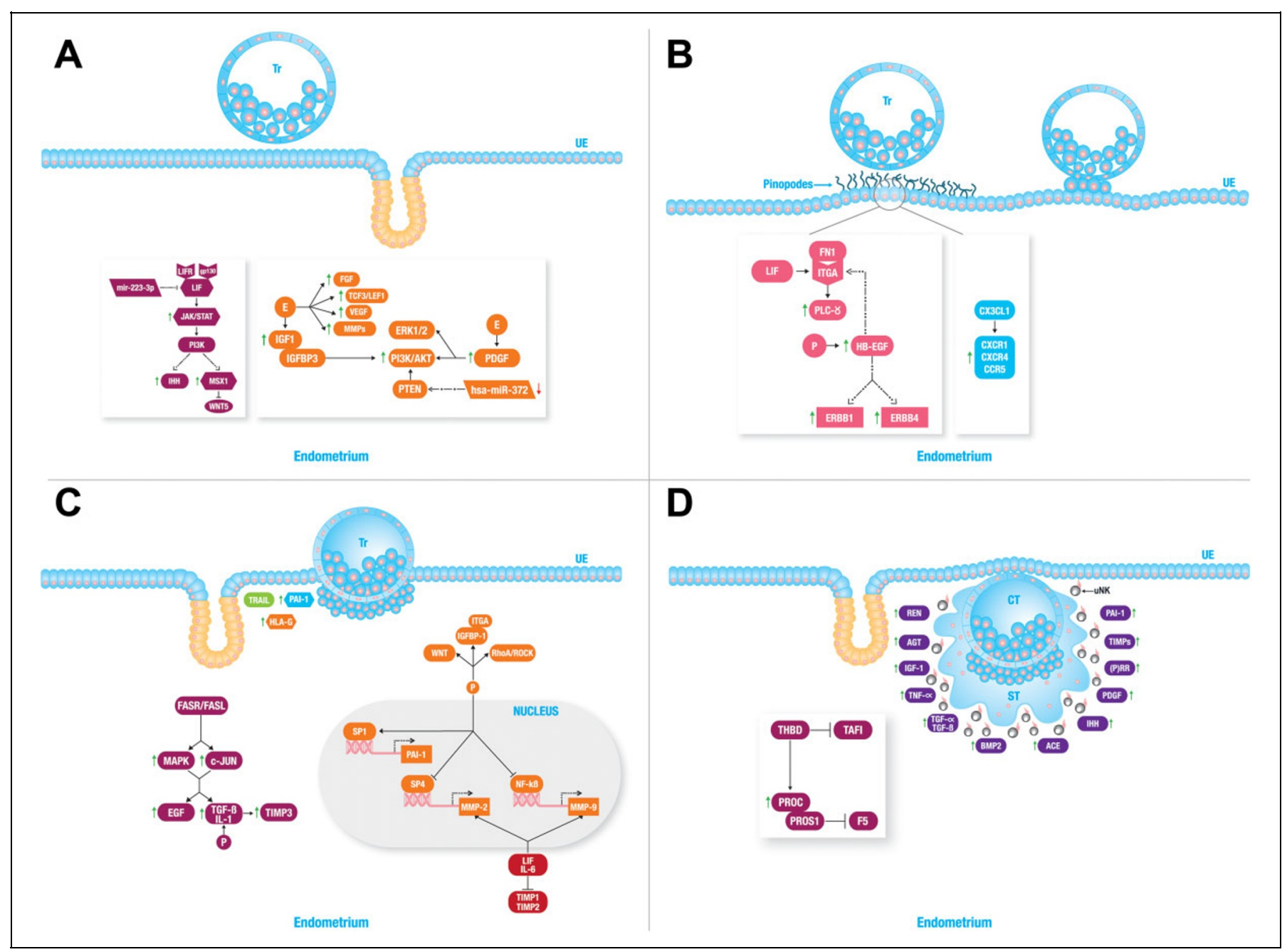

Figure I. Early RPL candidate genes. A, Uterine receptivity. Leukemia inhibitory factor and estrogen signaling during uterine receptivity in the endometrium's glandular epithelium for pinopode development, proliferation, differentiation, and cell survival. B, Apposition and adhesion. Integrin signaling in the luminal epithelium for implantation and chemokine upregulation in cell poles where the apposition will occur during apposition and adhesion. C, Apoptosis and invasion. FasR/FasL and progesterone signaling related to epithelial cell apoptosis. D, Decidualization and angiogenesis. Decidualization controls ST invasion, promotes placenta development, and remodels the endometrium's spiral arteries by REN, AGT, IGF-I, TNF- $\alpha$, TGF- $\alpha / \beta, B M P-2$, ACE, IHH, PDGF, (P)RR, TIMPs, and PAI-I upregulation and THBD signaling for blood flow hemostasis. The signaling pathway nomenclature follows Novère et al's guidelines published in $2009 .{ }^{87}$ RLP indicates recurrent pregnancy loss; Tr trophoblast; THBD, thrombomodulin; UE, uterine epithelium.

use as definitive clinically useful biomarkers. Furthermore, hundreds more genes may be coherent RPL candidate genes due to mammalian implantation's inherent complexity (Figure 1).

\section{Next-Generation Sequencing Technology}

\section{A Panoramic View of NGS Technology}

As mentioned beforehand, Sanger sequencing limitations mean that the simultaneous investigation of many variants throughout a genome is not feasible. PCR amplification and sequencing of multiple amplicons in large panels is technically complex, costly, and time-consuming.

Next-generation sequencing has thus emerged as a useful and efficient approach for analyzing large genomic regions at reasonable cost. Since whole-exome sequencing (WES) was first described in 2009, NGS has led to hundreds of new monogenic and polygenic disease-related genes and variants being identified, including those involved in reproductive disorders. ${ }^{27,28,61-63}$ Next-generation sequencing technology has also enabled large-scale sequencing of RNA, thereby facilitating de novo transcriptome sequence assembly, novel mutation identification (eg, splicing variants), and the determination of differential expression levels between conditions (eg, normal vs pathological). ${ }^{64,65}$

From a technical point of view, NGS has taken advantage of progress regarding microtechnology, enabling millions of bp to be analyzed in just a few hours using small chips, simple devices, and reliable experimental protocols. 
Whole-exome sequencing and target sequencing microarrays are frequently used for diagnostic and research purposes, while whole-genome sequencing is still exclusively reserved for research purposes. Significant levels of average read depth (the amount of times each nucleotide is read) must be ensured (especially for diagnostic applications) since NGS may involve a PCR step. It has been stated that $\sim 60 \times$ average depth is necessary for covering $>90 \%$ of target genes in WES experiments for research purposes, while up to $120 \times$ has been recommended for diagnosis. ${ }^{66,67}$ Trios (parental and proband DNA samples) have been used to establish numerous diseases' potential genetic origin, especially regarding monogenic disorders. However, its use is still limited for complex diseases (eg, RPL and other reproductive pathologies) because analyzing hundreds of variants to obtain (filter) coherent candidates is particularly challenging. ${ }^{28,68,69}$ This is particularly true for frequent disorders where numerous heterozygous sequence variants may contribute to the phenotype. Filtering is less difficult for rare recessive diseases affecting various patients belonging to the same family because biallelic variants rarely occur. ${ }^{70}$ However, families affected by reproductive disorders are uncommon due to negative selection.

Next-generation sequencing used for RNA molecule analysis has evolved broadly during the last few years, offering several advantages over classical expression microarray platforms, such as high dynamic range regarding expression assessment, insertion/deletion mapping, nucleotide change detection (including splice variants), determining transcript isoforms, and identifying gene fusions. ${ }^{65}$ RNAseq is used for research and diagnostic purposes, especially for understanding cancer's biological behavior and providing more accurate treatment. ${ }^{71,72}$ Next-generation sequencing has been used recently in single-cell genomics applications (eg, cancer research), thereby enhancing molecular/translational medicine development. ${ }^{73,74}$

\section{Next-Generation Sequencing Studies of Idiopathic RPL}

Attention has been focused in this section on studies aimed at describing maternal genetic factors (eg, endometrial) contributing to RPL; however, some reports regarding the fetal causes of RPL have been mentioned at the end of this section. At least 5 studies concerning idiopathic RPL women using NGS (DNA and RNA sequencing) have been described to date. ${ }^{29-32,34}$ It should be stressed that WES only detects single nucleotide variants and small indels; other genetic abnormalities are not covered by this approach, thereby limiting its usefulness for diagnosis.

Qiao et al have used WES regarding 7 euploid miscarriages from 4 families previously described as being affected by RPL. $^{29,75}$ The authors used unselected trio exome analysis to identify monogenic inheritance genes. Couples having suffered idiopathic RPL involving at least 2 miscarriage had been previously screened using array-CGH-detected CNVs. The DNA for WES analysis was extracted from miscarriage chorionic villi and parents' peripheral blood. Rare $(<1 \%$ minor allele frequencies [MAFs]) compound heterozygous variants were identified in 2 families in the $D Y N C 2 H 1$ (p.Tyr2016Cys and p.Asp2184Val) and ALOX15 genes (p.Tyr139Cys and p.Thr560Met). Data filtering, disease association enrichment analysis of variants, and software tools for predicting nonsynonymous variant functional implications were used to propose these sequence changes as potential RPL etiology candidates. Table 2 shows RPL patients' variants which have been screened by NGS.

DYNC2H1 is a cytoplasmic dynein protein involved in cilia synthesis and maintenance; mutant forms have been associated with lethal phenotypes in mice and humans. ${ }^{76-79} A L O X 15$ encodes an oxidizing enzyme which has been associated with inflammation, cancer and heart disease, and increased abortion rate in humans. ${ }^{80,81}$

Quintero-Ronderos et al have used a different approach, since WES was used for studying a panel of 49 Caucasian and Colombian unrelated women affected by idiopathic RPL. ${ }^{32}$ Contrary to the study by Qiao et al, other patients' family members were not included in this case study to filter potential etiological variants. These patients were screened for any known cause of RPL, and those having anomalies regarding karyotype, uterine anatomy, hormonal levels, prothrombotic factors, infections, and autoimmunity were excluded from the study. The DNA for the WES study was extracted from RPL patients' peripheral blood. ${ }^{32}$ Their bioinformatics analysis of data was focused on 234 RPL candidate genes. Stringent filters were used for selecting putative causative sequence variants: MAF $=0.00$, nonsynonymous variants, and changes in residues conserved during evolution. Twenty-seven variants affecting 22 genes were identified in $41 \%$ of the patients. These variants affected molecules involved in biological processes such as trophoblast/endometrium interaction, coagulation, angiogenesis, immunological function response/modulation, metabolism, ECM remodeling, steroidal nuclear receptor activation, and cell function regulation.

Some of these variants affected genes involved in molecular cascades and biological processes, that is, trophinin (TRO), $T H B D$, coagulation factor $\mathrm{V}(F 5)$, and leukemia inhibitory factor receptor alpha. Interestingly, adopting a robust quantum chemical approach (fragment molecular orbital analysis) for this study led to determining that the FGA-p.Phe685Cys and MMP10-p.Asp199Asn variants were related to substantial energetic changes, strongly suggesting a pathogenic effect and that they could be clinical biomarkers for RPL.

Concerning the NGS-RNAseq technique, 3 studies have recently used this approach for analyzing expression level disturbances in tissues involved in implantation failure and RPL. ${ }^{30,31,34}$ Sõber et al compared early placental chorionic villi transcriptomes and miRNomes from RPL Caucasian women having suffered at least 2 consecutive miscarriages to those from normal pregnancies (elective termination of pregnancy). Patients having known RPL risk factors were excluded, that is, abnormal menstrual cycle, genital infections, APS, thrombophilic variants, and an abnormal karyotype in the couple. Chorionic villi from cyto- and syncytiotrophoblasts were removed 


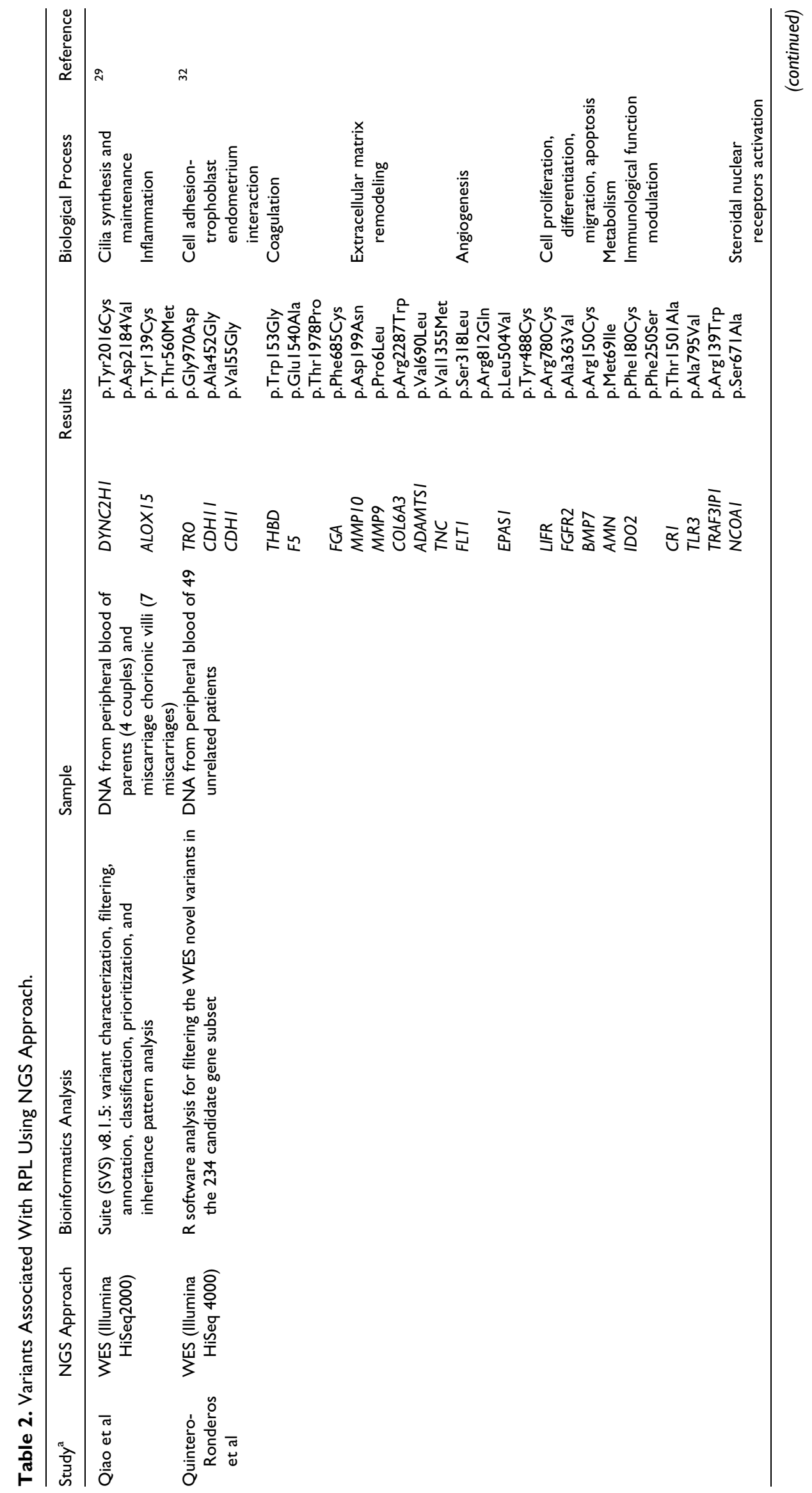




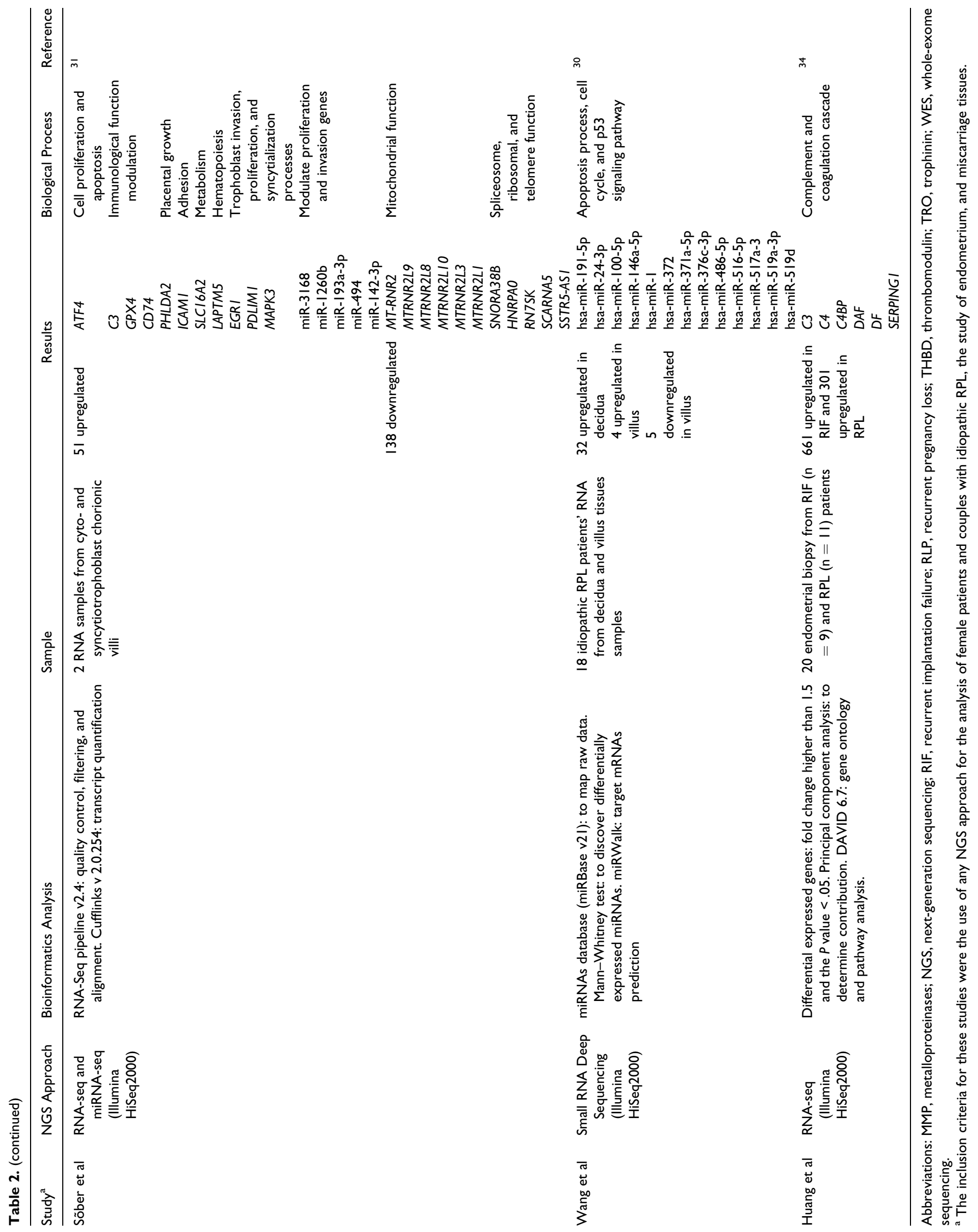


during surgical termination of the pregnancy for the RNA-Seq/ miRNA-Seq analysis. Two patients (5 and 6 RPL) were eligible for the RNA-Seq/miRNA-Seq study; 189 genes were found to be dysregulated between conditions (51 were upregulated and 138 downregulated). Upregulated molecules included ATF4, C3, PHLDA2, GPX4, ICAM1, and SLC16A2 which have been shown to play relevant roles during placental physiology and pregnancy complications. Downregulation of mitochondrial, spliceosome, ribosomal, and telomere function-related genes and intracellular signaling were identified. ${ }^{31}$

Furthermore, Wang et al have compared decidua and villus tissue miRNAomes from 18 Chinese RPL patients having a history of at least 2 consecutive pregnancy loses before the 12th week of gestation (confirmed by transvaginal ultrasound) to those from 15 normal pregnancies. ${ }^{30}$ Patients having classical risk factors were excluded, that is, abnormal parental karyotype, uterine anatomy, infectious and endocrine diseases, luteal phase alterations, and hyperprolactinemia. The decidua and villus tissues samples were collected by curettage from each patient. ${ }^{30}$ Thirty-two micro RNAs (miRNAs) were upregulated in decidua, while 4 were upregulated in RPL patients' villi. Only 5 miRNAs were found to be downregulated in RPL women's villi. The confirmed downregulated miRNAs were hsa-miR-1 and -372, which were associated with the increased expression of their predicted targets (BCL2 and PTEN, respectively).

More recently, Huang et al compared transcriptome data regarding endometrium samples from Chinese women affected by unexplained recurrent implantation failure (RIF) (defined as the failure to become pregnant after the transfer of at least 4 good embryos in a minimum of 3 cycles) to that from idiopathic RPL patients having had 3 or more consecutive miscarriages before the 24 th week of gestation. ${ }^{34}$ The patients included in the study were no more than 40 years old, with regular cycles, without a history of steroid hormone during the preceding 2 months. Having an abnormal karyotype, APS or systemic lupus erythematosus (SLE), abnormal thyroid function, uterine anomalies, intrauterine device in situ, and intrauterine adhesions were the exclusion criteria. Outpatient endometrial biopsy was performed on day luteinizing hormone $+7 . .^{34}$ More than 900 genes were dysregulated between conditions (661 upregulated in RIF and 301 upregulated in RPL). The complement and coagulation cascades (eg, $C 3, C 4, C 4 B P, D A F, D F$, and SERPING1 transcripts) were the main affected pathways.

It should be stressed that the aforementioned studies involved patients having idiopathic RPL. However, NGS has been reported as being a useful tool for identifying variants in genes related to rare disorders leading to RPL, such as IFT122, GLE1, and RYR 1. ${ }^{77,82}$ Next-generation sequencing has also helped to identify variants related to fetal molecular pathways in pregnancies having unexplained embryonic lethality or unexplained fetal malformations, regardless of family history. ${ }^{83-86}$ This approach thus helps screening, genetic diagnosis, and counselling regarding lethal fetal disorders in nonconsanguineous couples.
Taken together, NGS assays for research purposes have provided new insights into the biology of embryo implantation and pregnancy maintenance and enabled potential new molecular biomarkers for RPL diagnosis to be proposed in a clinical context.

\section{Sequencing Technologies for RPL Diagnosis and Future Directions}

Embryo implantation and pregnancy maintenance involve hundreds of genes acting in numerous molecular regulatory pathways; identifying mutations potentially related to RPL in such scenario is particularly difficult. Most studies have used Sanger's technique to analyze a limited number of genomic regions. This approach (in some cases complementing other genetic methods) has led to identifying sequence variants having restricted applications in a clinical environment. In fact, most studies have described sequence variants only having statistical associations with the phenotype, suggesting an increased risk of RPL. However, we consider that clinically useful molecular biomarkers should be validated by functional in vitro/in vivo tests. Genes having already published conclusive functional tests (eg, FOXD1, ALPP) may represent promising RPL diagnostic biomarkers since their missense mutations have been related to harmful effects. Regarding NGS, some commercial gene panels have been proposed for diagnostic purposes; however, most of them mainly include only coagulation molecules. Results must thus be interpreted with caution due to RPL's polygenic/multifactorial origin.

Furthermore, NGS at single-cell level should be used for identifying specific tissues' expressional signatures contributing to the phenotype. Such initiatives may enable a better understanding of RPL's pathophysiology, thus improving its molecular diagnosis. We consider that future therapeutics, including genome-editing procedures, will benefit from these approaches.

\section{Declaration of Conflicting Interests}

The author(s) declared no potential conflicts of interest with respect to the research, authorship, and/or publication of this article.

\section{Funding}

The author(s) disclosed receipt of the following financial support for the research, authorship, and/or publication of this article: The present study was financed by the Universidad del Rosario (grant CS/Genetics/ABN062-2018). Laissue's laboratory is funded by the Universidad del Rosario.

\section{References}

1. Mascarenhas MN, Flaxman SR, Boerma T, Vanderpoel S, Stevens GA. National, regional, and global trends in infertility prevalence since 1990: a systematic analysis of 277 health surveys. Low N, ed. PLoS Med. 2012;9(12):e1001356. doi:10.1371/journal.pmed.1001356. 
2. Datta J, Palmer MJ, Tanton C, et al. Prevalence of infertility and help seeking among 15000 women and men. Hum Reprod. 2016; 31(9):2108-2118. doi:10.1093/humrep/dew123.

3. Smith S, Pfeifer SM, Collins JA. Diagnosis and management of female infertility. JAMA. 2003;290(13):1767-1770. doi:10.1001/ jama.290.13.1767.

4. Teklenburg G, Salker M, Heijnen C, Macklon NS, Brosens JJ. The molecular basis of recurrent pregnancy loss: impaired natural embryo selection. Mol Hum Reprod. 2010;16(12):886-895. doi: 10.1093/molehr/gaq079.

5. van den Berg MM, van Maarle MC, van Wely M, Goddijn M. Genetics of early miscarriage. Biochim Biophys Acta. 2012; 1822(12):1951-1959. doi:10.1016/j.bbadis.2012.07.001.

6. Sugiura-Ogasawara M. Recurrent pregnancy loss and obesity. Best Pract Res Clin Obstet Gynaecol. 2015;29(4):489-497. doi: 10.1016/j.bpobgyn.2014.12.001.

7. Tur-Torres MH, Garrido-Gimenez C, Alijotas-Reig J. Genetics of recurrent miscarriage and fetal loss. Best Pract Res Clin Obstet Gynaecol. 2017;42:11-25. doi:10.1016/j.bpobgyn.2017.03.007.

8. Hogge WA, Byrnes AL, Lanasa MC, Surti U. The clinical use of karyotyping spontaneous abortions. Am J Obstet Gynecol. 2003; 189(2):397-400; discussion 400-402. doi:10.1067/S00029378(03)00700-2.

9. Rai R, Regan L. Recurrent miscarriage. Lancet. 2006;368(9535): 601-611. doi:10.1016/S0140-6736(06)69204-0.

10. Christiansen OB, Mathiesen O, Lauritsen JG, Grunnet N. Idiopathic recurrent spontaneous abortion. Evidence of a familial predisposition. Acta Obstet Gynecol Scand. 1990;69(7-8):597-601.

11. Christiansen OB, Pedersen B, Mathiesen O, Husth M, Grunnet N. Maternal HLA class II alleles predispose to pregnancy losses in Danish women with recurrent spontaneous abortions and their female relatives. Am J Reprod Immunol. 1996;35(3):239-244.

12. Kolte AM, Nielsen HS, Moltke I, et al. A genome-wide scan in affected sibling pairs with idiopathic recurrent miscarriage suggests genetic linkage. Mol Hum Reprod. 2011;17(6):379-385. doi: 10.1093/molehr/gar003.

13. Pereza N, Ostojić S, Kapović M, Peterlin B. Systematic review and meta-analysis of genetic association studies in idiopathic recurrent spontaneous abortion. Fertil Steril. 2017;107(1): 150-159 e2. doi:10.1016/j.fertnstert.2016.10.007.

14. Wang L, Wang ZC, Xie C, Liu XF, Yang MS. Genome-wide screening for risk loci of idiopathic recurrent miscarriage in a Han Chinese population: a pilot study. Reprod Sci. 2010;17(6): 578-584. doi:10.1177/1933719110364248.

15. Vaiman D. Genetic regulation of recurrent spontaneous abortion in humans. Biomed J. 2015;38(1):11-24. doi:10.4103/2319-4170. 133777.

16. Laissue P, Lakhal B, Benayoun BA, et al. Functional evidence implicating FOXL2 in non-syndromic premature ovarian failure and in the regulation of the transcription factor OSR2. $\mathrm{J} \mathrm{Med}$ Genet. 2009;46(7):455-457. doi:10.1136/jmg.2008.065086.

17. L'Hôte D, Laissue P, Serres C, Montagutelli X, Veitia RA, Vaiman D. Interspecific resources: a major tool for quantitative trait locus cloning and speciation research. Bioessays. 2010;32(2): 132-142. doi:10.1002/bies.200900027.
18. Vatin M, Burgio G, Renault G, et al. Refined mapping of a quantitative trait locus on chromosome 1 responsible for mouse embryonic death. Ebihara S, ed. PLoS One. 2012;7(8):e43356. doi:10.1371/journal.pone.0043356.

19. Vatin M, Bouvier S, Bellazi L, et al. Polymorphisms of human placental alkaline phosphatase are associated with in vitro fertilization success and recurrent pregnancy loss. Am J Pathol. 2014; 184(2):362-368. doi:10.1016/j.ajpath.2013.10.024.

20. Laissue P, Lakhal B, Vatin M, et al. Association of FOXD1 variants with adverse pregnancy outcomes in mice and humans. Open Biol. 2016;6(10) pii:160109. doi:10.1098/rsob.160109.

21. Stitziel NO, Kiezun A, Sunyaev S. Computational and statistical approaches to analyzing variants identified by exome sequencing. Genome Biol. 2011;12(9):227. doi:10.1186/gb-2011-12-9-227.

22. Koboldt DC, Steinberg KM, Larson DE, Wilson RK, Mardis ER. The next-generation sequencing revolution and its impact on genomics. Cell. 2013;155(1):27-38. doi:10.1016/j.cell.2013.09. 006.

23. McCarthy JJ, McLeod HL, Ginsburg GS. Genomic medicine: a decade of successes, challenges, and opportunities. Sci Transl Med. 2013;5(189):189sr4. doi:10.1126/scitranslmed.3005785.

24. Saare M, Rekker K, Laisk-Podar T, et al. High-throughput sequencing approach uncovers the miRNome of peritoneal endometriotic lesions and adjacent healthy tissues. Hawkins SM, ed. PLoS One. 2014;9(11):e112630. doi:10.1371/journal.pone. 0112630 .

25. Kaartokallio T, Cervera A, Kyllönen A, Laivuori K, Kere J, Laivuori H; FINNPEC Core Investigator Group. Gene expression profiling of pre-eclamptic placentae by RNA sequencing. Sci Rep. 2015;5:14107. doi:10.1038/srep14107.

26. Zhao L, Gu C, Ye M, et al. Identification of global transcriptome abnormalities and potential biomarkers in eutopic endometria of women with endometriosis: a preliminary study. Biomed Rep. 2017;6(6):654-662. doi:10.3892/br.2017.902.

27. Laissue P.Aetiological coding sequence variants in nonsyndromic premature ovarian failure: from genetic linkage analysis to next generation sequencing. Mol Cell Endocrinol. 2015; 411:243-257. doi:10.1016/j.mce.2015.05.005.

28. Laissue P. The molecular complexity of primary ovarian insufficiency aetiology and the use of massively parallel sequencing. Mol Cell Endocrinol. 2018;460:170-180. doi:10.1016/j.mce. 2017.07.021.

29. Qiao Y, Wen J, Tang F, et al. Whole exome sequencing in recurrent early pregnancy loss. Mol Hum Reprod. 2016;22(5):364-372. doi:10.1093/molehr/gaw008.

30. Wang JM, Gu Y, Zhang Y, et al. Deep-sequencing identification of differentially expressed miRNAs in decidua and villus of recurrent miscarriage patients. Arch Gynecol Obstet. 2016;293(5): 1125-1135. doi:10.1007/s00404-016-4038-5.

31. Sõber S, Rull K, Reiman M, Ilisson P, Mattila P, Laan M. RNA sequencing of chorionic villi from recurrent pregnancy loss patients reveals impaired function of basic nuclear and cellular machinery. Sci Rep. 2016;6(1):38439. doi:10.1038/srep38439.

32. Quintero-Ronderos P, Mercier E, Fukuda M, et al. Novel genes and mutations in patients affected by recurrent spontaneous abortion. PLoS One. 2017;12(10): 0186149. 
33. Tong J, Zhao W, Lv H, Li WP, Chen ZJ, Zhang C. Transcriptomic profiling in human decidua of severe preeclampsia detected by RNA sequencing. J Cell Biochem. 2018;119(1):607-615. doi:10. 1002/jcb.26221.

34. Huang J, Qin H, Yang Y, et al. A comparison of transcriptomic profiles in endometrium during window of implantation between women with unexplained recurrent implantation failure and recurrent miscarriage. Reproduction. 2017;153(6):749-758. doi:10. 1530/REP-16-0574.

35. Norwitz ER, Snegovskikh V, Schatz F, et al. Progestin inhibits and thrombin stimulates the plasminogen activator/inhibitor system in term decidual stromal cells: implications for parturition. Am J Obstet Gynecol. 2007;196(4):382.e1-382.e8. doi:10.1016/j. ajog.2007.02.035.

36. Lumbers ER, Wang Y, Delforce SJ, et al. Decidualisation of human endometrial stromal cells is associated with increased expression and secretion of prorenin. Reprod Biol Endocrinol. 2015;13(1):129. doi:10.1186/s12958-015-0127-8.

37. Mary MJ, Saravanan L, Deecaraman M, Vijayalakshmi M, Umashankar V, Sailaja J. Polymorphism of the PAI-1gene (4G/5G) may be linked with polycystic ovary syndrome and associated pregnancy disorders in South Indian Women. Bioinformation. 2017;13(5):149-153. doi:10.6026/97320630013149.

38. Pfeiffer KA, Fimmers R, Engels G, van der Ven H, van der Ven $\mathrm{K}$. The HLA-G genotype is potentially associated with idiopathic recurrent spontaneous abortion. Mol Hum Reprod. 2001;7(4): 373-378.

39. Agrawal D, Prakash S, Misra MK, Phadke SR, Agrawal S. Implication of HLA-G $5^{\prime}$ upstream regulatory region polymorphisms in idiopathic recurrent spontaneous abortions. Reprod Biomed Online. 2015;30(1):82-91. doi:10.1016/j.rbmo.2014.09.015.

40. Nardi Fda S, Slowik R, Wowk PF, et al. Analysis of HLA-G polymorphisms in couples with implantation failure. Am J Reprod Immunol. 2012;68(6):507-514. doi:10.1111/aji.12001.

41. Hviid TV, Hylenius S, Hoegh AM, Kruse C, Christiansen OB. HLA-G polymorphisms in couples with recurrent spontaneous abortions. Tissue Antigens. 2002;60(2):122-132. doi:10.1034/j. 1399-0039.2002.600202.x.

42. Mohlin FC, Mercier E, Fremeaux-Bacchi V, et al. Analysis of genes coding for CD46, CD55, and C4b-binding protein in patients with idiopathic, recurrent, spontaneous pregnancy loss. Eur J Immunol. 2013;43(6):1617-1629. doi:10.1002/eji. 201243196.

43. Sun Q, Zhang XL. Research on apoptotic signaling pathways of recurrent spontaneous abortion caused by dysfunction of trophoblast infiltration. Eur Rev Med Pharmacol Sci. 2017;21(suppl 3): 12-19.

44. Mohammad Seyedhassani S, Houshmand M, Kalantar SM, et al. BAX pro-apoptotic gene alterations in repeated pregnancy loss. Arch Med Sci. 2011;7(1):117-122. doi:10.5114/aoms.2011. 20614.

45. Halasz M, Szekeres-Bartho J. The role of progesterone in implantation and trophoblast invasion. J Reprod Immunol. 2013;97(1): 43-50. doi:10.1016/j.jri.2012.10.011.

46. Tang C, Mei L, Pan L, et al. Hedgehog signaling through GLI1 and GLI2 is required for epithelial-mesenchymal transition in human trophoblasts. Biochim Biophys Acta. 2015;1850(7): 1438-1448. doi:10.1016/j.bbagen.2015.04.005.

47. DaSilva-Arnold S, James JL, Al-Khan A, Zamudio S, Illsley NP. Differentiation of first trimester cytotrophoblast to extravillous trophoblast involves an epithelial-mesenchymal transition. Placenta. 2015;36(12):1412-1418. doi:10.1016/j.placenta.2015.10. 013.

48. Staun-Ram E, Goldman S, Gabarin D, Shalev E. Expression and importance of matrix metalloproteinase 2 and 9 (MMP-2 and -9) in human trophoblast invasion. Reprod Biol Endocrinol. 2004; 2(1):59. doi:10.1186/1477-7827-2-59.

49. Shimonovitz S, Hurwitz A, Hochner-Celnikier D, Dushnik M, Anteby E, Yagel S. Expression of gelatinase B by trophoblast cells: down-regulation by progesterone. Am J Obstet Gynecol. 1998;178(3):457-461.

50. Higuchi T, Kanzaki H, Nakayama H, et al. Induction of tissue inhibitor of metalloproteinase 3 gene expression during in vitro decidualization of human endometrial stromal cells. Endocrinology. 1995;136(11):4973-4981. doi:10.1210/endo.136.11. 7588231.

51. Imakawa K, Bai R, Fujiwara H, Ideta A, Aoyagi Y, Kusama K. Continuous model of conceptus implantation to the maternal endometrium. J Endocrinol. 2017;233(1):R53-R65. doi:10. 1530/JOE-16-0490.

52. Goldman S, Lovett DH, Shalev E. Mechanisms of matrix metalloproteinase-2 (mmp-2) transcriptional repression by progesterone in jar choriocarcinoma cells. Reprod Biol Endocrinol. 2009;7(1):41. doi:10.1186/1477-7827-7-41.

53. Song G, Yan J, Zhang Q, Li G, Chen ZJ. Association of tissue inhibitor of metalloproteinase gene polymorphisms and unexplained recurrent spontaneous abortions in Han Chinese couples. Eur J Obstet Gynecol Reprod Biol. 2014;181:84-88. doi:10.1016/ j.ejogrb.2014.07.013.

54. Sherer DM, Abulafia O. Angiogenesis during implantation, and placental and early embryonic development. Placenta. 2001; 22(1):1-13. doi:10.1053/plac.2000.0588.

55. Boeddeker SJ, Hess AP. The role of apoptosis in human embryo implantation. J Reprod Immunol. 2015;108:114-122. doi:10. 1016/j.jri.2015.02.002.

56. Kaare M, Ulander VM, Painter JN, Ahvenainen T, Kaaja R, Aittomäki K. Variations in the thrombomodulin and endothelial protein $\mathrm{C}$ receptor genes in couples with recurrent miscarriage. Hum Reprod. 2007;22(3):864-868. doi:10.1093/humrep/del436.

57. Quintero-Ronderos P, Mercier E, Gris JC, et al. THBD sequence variants potentially related to recurrent pregnancy loss. Reprod Biol Endocrinol. 2017;15(1):92. doi:10.1186/s12958-017-0311-0.

58. Ticconi C, Mancinelli F, Gravina P, Federici G, Piccione E, Bernardini S. Beta-fibrinogen G-455A polymorphisms and recurrent miscarriage. Gynecol Obstet Invest. 2011;71(3):198-201. doi:10. $1159 / 000317522$.

59. Masini S, Ticconi C, Gravina P, et al. Thrombin-activatable fibrinolysis inhibitor polymorphisms and recurrent pregnancy loss. Fertil Steril. 2009;92(2):694-702. doi:10.1016/j.fertnstert.2008. 07.015 .

60. Kaare M, Painter JN, Ulander VM, Kaaja R, Aittomäki K. Variations of the amnionless gene in recurrent spontaneous abortions. 
Mol Hum Reprod. 2006;12(1):25-29. doi:10.1093/molehr/ gah255.

61. Ng SB, Turner EH, Robertson PD, et al. Targeted capture and massively parallel sequencing of 12 human exomes. Nature. 2009:461(7261):272-276. doi:10.1038/nature08250.

62. Ezewudo M, Zwick ME. Evaluating rare variants in complex disorders using next-generation sequencing. Curr Psychiatry Rep. 2013;15(4):349. doi:10.1007/s11920-013-0349-4.

63. Stranneheim H, Wedell A. Exome and genome sequencing: a revolution for the discovery and diagnosis of monogenic disorders. J Intern Med. 2016;279(1):3-15. doi:10.1111/joim.12399.

64. Han Y, Gao S, Muegge K, Zhang W, Zhou B. Advanced applications of RNA sequencing and challenges. Bioinform Biol Insights. 2015;9(suppl 1):29-46. doi:10.4137/BBI.S28991.

65. Byron SA, Van Keuren-Jensen KR, Engelthaler DM, Carpten JD, Craig DW. Translating RNA sequencing into clinical diagnostics: opportunities and challenges. Nat Rev Genet. 2016;17(5): 257-271. doi:10.1038/nrg.2016.10.

66. Sims D, Sudbery I, Ilott NE, Heger A, Ponting CP. Sequencing depth and coverage: key considerations in genomic analyses. Nat Rev Genet. 2014;15(2):121-132. doi:10.1038/nrg3642.

67. Kim K, Seong MW, Chung WH, et al. Effect of next-generation exome sequencing depth for discovery of diagnostic variants. Genomics Inform. 2015;13(2):31-39. doi:10.5808/GI.2015.13.2. 31.

68. Patiño LC, Beau I, Carlosama C, et al. New mutations in nonsyndromic primary ovarian insufficiency patients identified via whole-exome sequencing. Hum Reprod. 2017;32(7):1512-1520. doi:10.1093/humrep/dex089.

69. Quintero-Ronderos P, Mercier E, Fukuda M, et al. Novel genes and mutations in patients affected by recurrent pregnancy loss. Bandapalli OR, ed. PLoS One. 2017;12(10):e0186149. doi:10. 1371/journal.pone.0186149.

70. Carlosama C, Elzaiat M, Patiño LC, Mateus HE, Veitia RA, Laissue P. A homozygous donor splice-site mutation in the meiotic gene MSH4 causes primary ovarian insufficiency. Hum Mol Genet. 2017;26(16):3161-3166. doi:10.1093/hmg/ddx199.

71. Costa V, Aprile M, Esposito R, Ciccodicola A. RNA-Seq and human complex diseases: recent accomplishments and future perspectives. Eur J Hum Genet. 2013;21(2):134-142. doi:10.1038/ ejhg.2012.129.

72. LeBlanc VG, Marra MA. Next-generation sequencing approaches in cancer: where have they brought us and where will they take us? Cancers (Basel). 2015;7(3):1925-1958. doi:10.3390/ cancers7030869.

73. Müller S, Diaz A. Single-cell mRNA sequencing in cancer research: integrating the genomic fingerprint. Front Genet. 2017;8:73. doi:10.3389/fgene.2017.00073.
74. Tsoucas D, Yuan GC. Recent progress in single-cell cancer genomics. Curr Opin Genet Dev. 2017;42:22-32. doi:10.1016/j.gde. 2017.01.002.

75. Rajcan-Separovic E, Diego-Alvarez D, Robinson WP, et al. Identification of copy number variants in miscarriages from couples with idiopathic recurrent pregnancy loss. Hum Reprod. 2010; 25(11):2913-2922. doi:10.1093/humrep/deq202.

76. Grissom PM, Vaisberg EA, McIntosh JR. Identification of a novel light intermediate chain (D2LIC) for mammalian cytoplasmic dynein 2. Mol Biol Cell. 2002;13(3):817-829. doi:10.1091/mbc.01-08-0402.

77. Ellard S, Kivuva E, Turnpenny P, et al. An exome sequencing strategy to diagnose lethal autosomal recessive disorders. Eur $J$ Hum Genet. 2015;23(3):401-404. doi:10.1038/ejhg.2014.120.

78. Huangfu D, Anderson K V. Cilia and Hedgehog responsiveness in the mouse. Proc Natl Acad Sci US A. 2005;102(32): 11325-11330. doi:10.1073/pnas.0505328102.

79. May SR, Ashique AM, Karlen M, et al. Loss of the retrograde motor for IFT disrupts localization of Smo to cilia and prevents the expression of both activator and repressor functions of Gli. Dev Biol. 2005;287(2):378-389. doi:10.1016/j.ydbio.2005.08.050.

80. Ivanov I, Kuhn H, Heydeck D. Structural and functional biology of arachidonic acid 15-lipoxygenase-1 (ALOX15). Gene. 2015; 573(1):1-32. doi:10.1016/j.gene.2015.07.073.

81. Dar P, Strassburger D, Shaish A, Levkovitz H, Halperin R, Harats D. Reduced reproduction with increased abortion rate in transgenic mice that overexpress 15-lipoxygenase. Gynecol Obstet Invest. 2001;52(1):18-21. doi:10.1159/000052934.

82. Tsurusaki Y, Yonezawa R, Furuya M, et al. Whole exome sequencing revealed biallelic IFT122 mutations in a family with CED1 and recurrent pregnancy loss. Clin Genet. 2014;85(6):592-594. doi:10.1111/cge.12215.

83. Shamseldin HE, Kurdi W, Almusafri F, et al. Molecular autopsy in maternal-fetal medicine. Genet Med. 2018;20(4):420-427. doi: 10.1038/gim.2017.111.

84. Shamseldin HE, Tulbah M, Kurdi W, et al. Identification of embryonic lethal genes in humans by autozygosity mapping and exome sequencing in consanguineous families. Genome Biol. 2015;16(1):116. doi:10.1186/s13059-015-0681-6.

85. Filges I, Friedman JM. Exome sequencing for gene discovery in lethal fetal disorders - harnessing the value of extreme phenotypes. Prenat Diagn. 2015;35(10):1005-1009. doi:10.1002/pd. 4464.

86. Filges I, Nosova E, Bruder E, et al. Exome sequencing identifies mutations in KIF14 as a novel cause of an autosomal recessive lethal fetal ciliopathy phenotype. Clin Genet. 2014;86(3): 220-228. doi:10.1111/cge.12301.

87. Le Novère $\mathrm{N}$, Hucka $\mathrm{M}$, Mi H, et al. The systems biology graphical notation. Nat Biotechnol. 2009;27(8):735-741. doi:10. 1038/nbt. 1558 . 